EPJ Web of Conferences 71, 00098 (2014)

DOI: 10.1051/epjconf/20147100098

(C) Owned by the authors, published by EDP Sciences, 2014

\title{
Heavy flavors in nucleus-nucleus collisions at RHIC and LHC
}

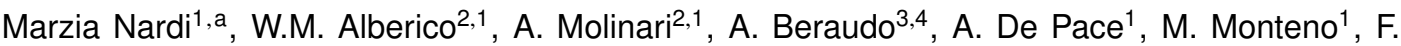 \\ Prino ${ }^{1}$, and M. Sitta ${ }^{5,1}$ \\ ${ }^{1}$ INFN, Sezione di Torino, Italy \\ ${ }^{2}$ Dip.to di Fisica, Univ. Torino, Italy \\ ${ }^{3}$ Physics Department, Theory Unit, CERN, Switzerland \\ ${ }^{4}$ Dep. de Fisica de Particulas, Univ. Santiago de Compostela, Spain \\ ${ }^{5}$ Univ. Piemonte Orientale, Alessandria, Italy
}

\begin{abstract}
A multi-step setup for heavy-flavor studies in high-energy nucleus-nucleus $(A A)$ collisions - addressing within a comprehensive framework the initial $Q \bar{Q}$ production, the propagation in the hot medium until decoupling and the final hadronization and decays - is presented. The propagation of the heavy quarks in the medium is described in a framework provided by the relativistic Langevin equation and the corresponding numerical results are compared to experimental data from RHIC and the LHC. In particular, outcomes for the nuclear modification factor $R_{A A}$ and for the elliptic flow $v_{2}$ of $D / B$ mesons, heavy-flavor electrons and non-prompt $J / \psi$ 's are displayed.
\end{abstract}

\section{Introduction}

The purpose of our work is to provide a comprehensive setup for the study of heavy-flavor observables in high-energy hadronic ( $p p$ ) and nuclear $(A A)$ collisions, from the $Q \bar{Q}$ production, in hard nucleonnucleon collisions, to the detection in the experimental apparatus.

The interest in heavy quarks for heavy-ion phenomenology lies in the fact that, being produced in the first instants, they allow a tomography of the medium formed in high-energy $A A$ collisions, the Quark Gluon Plasma (QGP). In fact, because of the large mass, their initial production is a shortdistance process described by $\mathrm{pQCD}$. Hence, differences in the final observables with respect to the $p p$ and $p A$ benchmark reflect the presence of a dense medium formed in the collision and allow us to test its properties.

\section{Proton-proton collisions: the baseline}

Although the main goal of our work is to study medium effects on heavy-flavor observables in $A A$ collisions, one needs first of all to validate the tools employed in simulating the initial $Q \bar{Q}$ production through a comparison with the experimental data collected in $p p$ collisions. For this purpose we rely on a standard pQCD public tool, namely POWHEG-BOX, in which the hard $Q \bar{Q}$ event (under control, due to the large quark mass) is interfaced with a shower stage described with PYTHIA, to include the

a e-mail: nardi@to.infn.it

This is an Open Access article distributed under the terms of the Creative Commons Attribution License 2.0, which permits unrestricted use, distribution, and reproduction in any medium, provided the original work is properly cited. 
effects of Initial State Radiation (ISR) and Final State Radiation (FSR), re-summing multiple emission of soft/collinear gluons at Leading Log (LL) accuracy. Intrinsic- $k_{T}$ corrections (with $\left\langle k_{T}^{2}\right\rangle=1 \mathrm{GeV}^{2}$ ) are also included in the simulation of the heavy-quark production.

Concerning the hadronization stage we adopt essentially the same fragmentation setup employed by FONLL, which was tuned by the authors to reproduce experimental $e^{+} e^{-}$data. Heavy quarks are made hadronize by sampling different hadron species from $c$ and $b$ fragmentation fractions extracted from experimental data [13-15]. Then, hadron momenta are sampled from Fragmentation Functions (FFs). The parametrization used in our work is taken from the literature [16, 17], with the phenomenological parameters fitted to reproduce experimental data [14, 18, 19]

The agreement between our results and the experimental data, on $c \bar{c}$ and $b \bar{b}$ production, provided by ALICE and CMS Collaborations in $p p$ collisions at $\sqrt{s}=2.76$ and $7 \mathrm{TeV}$ is fairly good [3].

\section{Nucleus-Nucleus collisions}

\subsection{Heavy-quark Production}

The main tool to describe the $c \bar{c}$ or $b \bar{b}$ production in a collision between heavy nuclei is again POWHEG-BOX, as in the $p p$ case, but with two important differences.

First of all, the nuclear PDF's should be modified by including nuclear corrections: we have adopted here the EPS09 scheme [7]. In principle, the density probed by the colliding partons should depend on the impact parameter $b$ : in describing nucleus-nucleus collisions we have made the simple choice of employing the EPS09 scheme for impact parameters $b<2 R$ and of neglecting nuclear corrections for $b>2 R$ ( $R$ being the radius of the nuclear density distribution).

A second difference one has to cope with in $A A$ collisions concerns the larger transverse momentum acquired on average by the colliding partons, because of the large size of the traversed medium. To get a realistic estimate for $\left\langle k_{T}^{2}\right\rangle_{A A}$ in nucleus-nucleus collisions we have adopted a Glauber approach [2]. One gets an average squared transverse momentum that depends not only on the impact parameter of the collision and on the nuclei involved, but also on the position of the $Q \bar{Q}$ pair in the transverse plane.

\subsection{Heavy quarks in the medium}

The medium produced in $\mathrm{Au}-\mathrm{Au}$ and $\mathrm{Pb}-\mathrm{Pb}$ collisions is described through hydrodynamical calculations performed with the viscous $2+1$ code of Ref. [10], using Glauber initial conditions with $\sigma_{N N}=42 \mathrm{mb}$ and $\sigma_{N N}=64 \mathrm{mb}$ for RHIC and the LHC, respectively. The assumption of longitudinal invariance (implicit in the $2+1$ description) restricts the validity of our calculation in a limited region around mid-rapidity.

The propagation of the heavy quarks in the plasma is modeled as a Brownian motion by employing a relativistic Langevin equation:

$$
\frac{d \vec{p}}{d t}=-\eta_{D}(p) \vec{p}+\vec{\xi}(t)
$$

where the drag coefficient $\eta_{D}(p)$ describes the deterministic friction force acting on the heavy quark, whereas the term $\vec{\xi}$ accounts for the random collisions with the constituents of the medium. The effect of the stochastic term is completely determined once its temporal correlation function is fixed. The latter is usually assumed to be given by

$$
\left\langle\xi^{i}(t) \xi^{j}\left(t^{\prime}\right)\right\rangle=b^{i j}(\vec{p}) \delta\left(t-t^{\prime}\right)
$$


entailing that collisions at different time-steps are uncorrelated. The tensor $b^{i j}(\vec{p})$ can be decomposed with a standard procedure according to

$$
b^{i j}(\vec{p}) \equiv \kappa_{L}(p) \hat{p}^{i} \hat{p}^{j}+\kappa_{T}(p)\left(\delta^{i j}-\hat{p}^{i} \hat{p}^{j}\right)
$$

in terms of the coefficients $\kappa_{L / T}(p)$, which represent the squared longitudinal/transverse momentum per unit time exchanged by the quark with the medium. It is useful to introduce the related tensor

$$
g^{i j}(\vec{p}) \equiv \sqrt{\kappa_{L}(p)} \hat{p}^{i} \hat{p}^{j}+\sqrt{\kappa_{T}(p)}\left(\delta^{i j}-\hat{p}^{i} \hat{p}^{j}\right) \equiv g_{L}(p) \hat{p}^{i} \hat{p}^{j}+g_{T}(p)\left(\delta^{i j}-\hat{p}^{i} \hat{p}^{j}\right),
$$

which allows one to factor out the momentum dependence of the noise term in Eq. (1), thus obtaining the equation

$$
\frac{d p^{i}}{d t}=-\eta_{D}(p) p^{i}+g^{i j}(\vec{p}) \eta^{j}(t)
$$

with

$$
\left\langle\eta^{i}(t) \eta^{j}\left(t^{\prime}\right)\right\rangle=\delta^{i j} \delta\left(t-t^{\prime}\right)
$$

The set of Eqs. (4-6) is defined in the rest frame of the background medium and it allows one to study the quark propagation once the transport coefficients are given. These depend on the medium temperature, which in turn, in the expanding fireball, depends on the space-time position occupied by the heavy quark.

At each time-step (assumed, in our simulation, to be $0.02 \mathrm{fm} / c$ ), we make a Lorentz transformation to the fluid local rest frame, update the heavy-quark momentum according to the above equations (the three components $\eta_{i}$ are sampled randomly and independently), and boost back to the lab-frame: this makes the heavy quark acquire (part of) the flow of the medium.

Heavy quark transport coefficients can be evaluated starting from their definition

$$
\kappa_{L}=\left\langle\frac{\Delta \mathbf{q}_{L}^{2}}{\Delta t}\right\rangle \quad \text { and } \quad \kappa_{T}=\frac{1}{2}\left\langle\frac{\Delta \mathbf{q}_{T}^{2}}{\Delta t}\right\rangle .
$$

The momentum broadening (and degradation) of heavy quarks in the medium must arise from their interaction with the other constituents of the plasma: light quarks and gluons. Within a perturbative setup, if the four-momentum exchange is sufficiently hard $\left(|t|>|t|^{*}\right.$, where $\left.t \equiv \omega^{2}-\mathbf{q}^{2}\right)$ one is dealing with a short-distance process and the result is given by a kinetic pQCD calculation:

$$
\kappa_{L, \text { hard }}^{g / q}=\frac{1}{2 E} \int_{k} \frac{n_{B / F}(k)}{2 k} \int_{k^{\prime}} \frac{1 \pm n_{B / F}\left(k^{\prime}\right)}{2 k^{\prime}} \int_{p^{\prime}} \frac{\theta\left(|t|-|t|^{*}\right)}{2 E^{\prime}}(2 \pi)^{4} \delta^{(4)}\left(P+K-P^{\prime}-K^{\prime}\right)\left|\overline{\mathcal{M}}_{g / q}(s, t)\right|^{2} \mathbf{q}_{L}^{2}
$$

and

$$
\kappa_{T, \text { hard }}^{g / q}=\frac{1}{2 E} \int_{k} \frac{n_{B / F}(k)}{2 k} \int_{k^{\prime}} \frac{1 \pm n_{B / F}\left(k^{\prime}\right)}{2 k^{\prime}} \int_{p^{\prime}} \frac{\theta\left(|t|-|t|^{*}\right)}{2 E^{\prime}}(2 \pi)^{4} \delta^{(4)}\left(P+K-P^{\prime}-K^{\prime}\right)\left|\overline{\mathcal{M}}_{g / q}(s, t)\right|^{2} \frac{\mathbf{q}_{T}^{2}}{2} .
$$

If, on the contrary, the momentum transfer is soft $\left(|t|<|t|^{*}\right)$, the scattering involves the exchange of a long wavelength gluon, which requires the resummation of medium effects. This can be done in hotQCD within the Hard Thermal Loop approximation. The corresponding contribution to $\kappa_{L / T}$ has been derived and evaluated in Refs. [1,2]. Eventually, one has to sum-up the soft and hard contributions to the transport coefficients :

$$
\kappa_{L / T}=\kappa_{L / T}^{\mathrm{soft}}+\kappa_{L / T}^{\mathrm{hard}}
$$

The scale of the strong coupling constant $g$ has been set at the typical thermal momentum, $\mu \sim T$, in the 

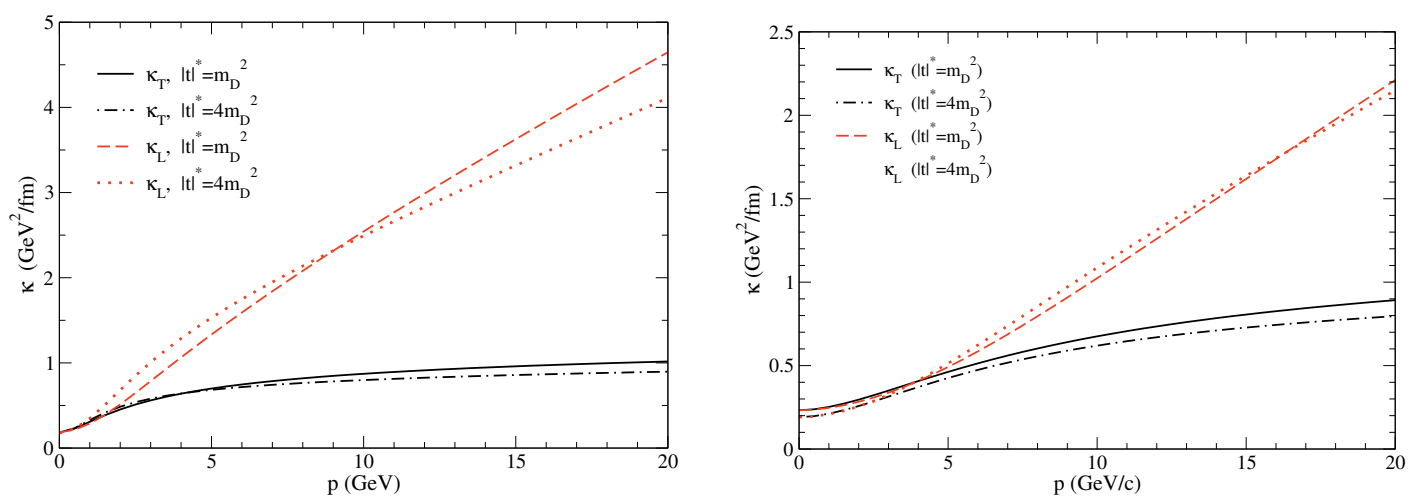

Figure 1. Transport coefficients for $c$ (left panel) and $b$ (right panel) quarks in the QGP. The curves refer to the temperature $T=400 \mathrm{MeV}$ and the running coupling has been evaluated at the scale $\mu=1.5 \pi T$ in the soft contribution and at the scale $\mu=\sqrt{|t|}$, set by the four-momentum exchange, in the calculation of the hard collisions.

soft contribution and to the squared-momentum transfer in the collisions, $\mu \sim \sqrt{|t|}$, in the evaluation of $\kappa^{\text {hard }}$. Fig. 1 summarizes the essential features of the results for the heavy-quark transport coefficients. First of all we note that, choosing $|t|^{*} \sim m_{D}^{2}$ (the Debye-mass $m_{D}$ being responsible for the screening of electric fields in the plasma), the dependence of $\kappa_{L / T}$ on the unphysical intermediate cutoff $|t|^{*}$ is very mild. The transverse coefficients saturate at high $p_{T}$, while the longitudinal ones show a rapid increase (especially for the $c$ quark): this fact has relevant consequences on the physical observables.

Finally, after the propagation through the dense medium, the heavy quarks decouple and hadronize. In this stage we adopt the same procedure used in the $p p$ case, neglecting the possibility of recombination (coalescence) of the final heavy quarks with light quarks of the medium. While this can be justified for high $p_{T}$ 's, the coalescence may play an important role in the low $p_{T}$ region, as we shall discuss in the following.

\section{Results}

This section will present a selection of our results, compared with experimental data obtained at RHIC and LHC energies.

In Fig. 2 we display the outcomes of our Langevin setup for the nuclear modification factors $R_{A A}\left(p_{T}\right)$ of $D^{0}$ mesons in central and minimum-bias Au-Au collisions at RHIC, compared to preliminary STAR data [20]. The size of the suppression for $p_{T} \gtrsim 2 \mathrm{GeV}$ in central $(0-10 \%)$ events is quite well reproduced by the HTL curve. On the other hand experimental data display a bump around $p_{T} \sim 1.5 \mathrm{GeV} / \mathrm{c}$, with $R_{A A}>1$ in the $p_{T}$ range $1-2 \mathrm{GeV} / \mathrm{c}$ and a depletion at smaller $p_{T}$, which is missed by our model. Such a behavior at low $p_{T}$ (say, for $p_{T} \leqslant 3 \mathrm{GeV} / \mathrm{c}$ ) might come from coalescence [21], so far not implemented into our framework.

In Fig. 3 we compare PHENIX [22] data and the preliminary STAR data for non-photonic electrons [23], to our Langevin outcomes for heavy flavor decay electrons $\left(e_{c+b}\right)$ in Au-Au collisions for different centrality classes. Theory outcomes nicely reproduce the data for large enough $p_{T}$ (say, $p_{T} \gtrsim 4 \mathrm{GeV} / \mathrm{c}$ ), missing the enhancement observed in the low-momentum region.

In Fig. 4 we display our results for the $D$-meson $R_{A A}$, as a function of the centrality, in $\mathrm{Pb}-\mathrm{Pb}$ collisions at the LHC compared to ALICE data [24]. HTL transport coefficients reproduce quite 


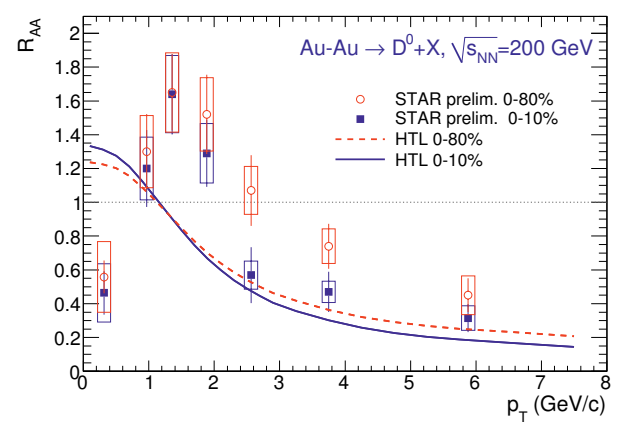

Figure 2. Results for the $R_{A A}$ of $D^{0}$ mesons in central $(0-10 \%$, in blue, solid line $)$ and minimum-bias $(0-80 \%$, in red, dashed line $) \mathrm{Au}-\mathrm{Au}$ collisions at $\sqrt{s_{N N}}=200 \mathrm{GeV}$ compared to preliminary STAR data [20].

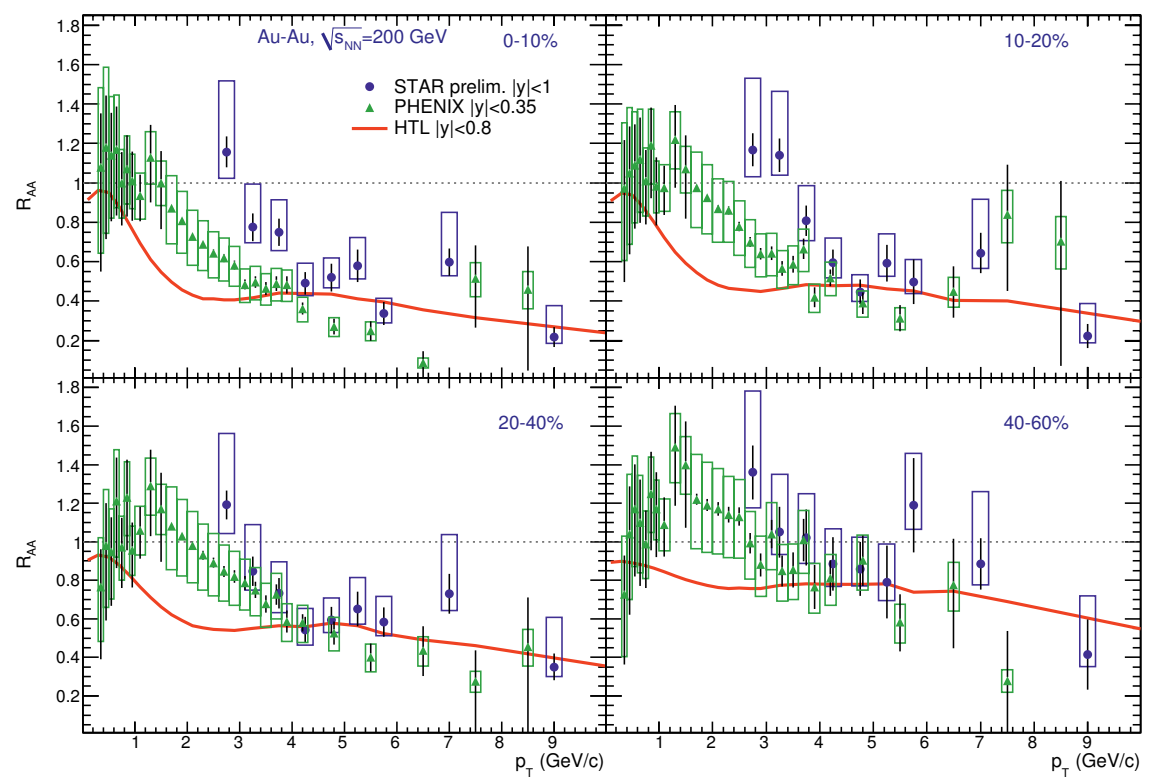

Figure 3. Results (with HTL transport coefficients) for the $R_{A A}$ of non-photonic electrons $\left(e_{c+b}\right)$ from charm and beauty decays in Au-Au collisions at $\sqrt{s_{N N}}=200 \mathrm{GeV}$ for various centrality classes compared to PHENIX [22] and preliminary STAR [23] data.

nicely the data at moderate $p_{T}$, but at larger $p_{T}$ they would entail a too strong quenching of the spectra, presumably due to the rapid rise of $\kappa_{L}(p)$ with the quark momentum.

Preliminary ALICE results [25] on electrons from charm and beauty decays have also become available; we display them in Fig. 5 compared with the outcomes of our Langevin simulations for $R_{A A}$ in central events. The size of the suppression is quite well reproduced.

Let us now consider the azimuthal anisotropy of the momenta of the heavy flavor hadrons produced in the collision and of their decay electrons. The anisotropy is characterized by the Fourier coefficients $v_{n}=\left\langle\cos \left[n\left(\varphi-\Psi_{\mathrm{RP}}\right)\right]\right\rangle$, where $\varphi$ is the particle azimuthal angle and $\Psi_{\mathrm{RP}}$ is the azimuthal 

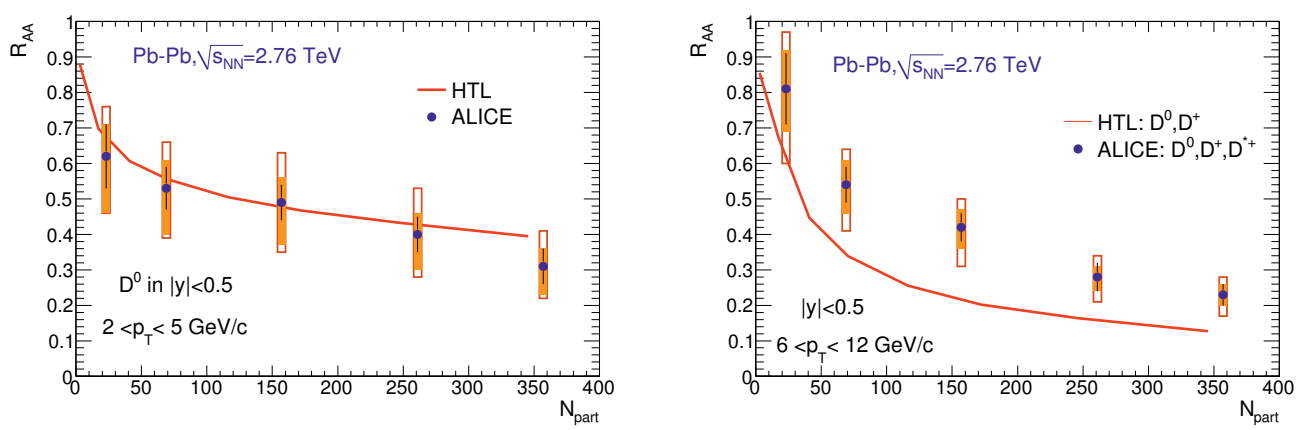

Figure 4. Centrality dependence of the $D$-meson $R_{A A}$ in $\mathrm{Pb}-\mathrm{Pb}$ collisions. Results with different transport coefficients are compared to ALICE data [24] at moderate (left panel) and large (right panel) momenta.

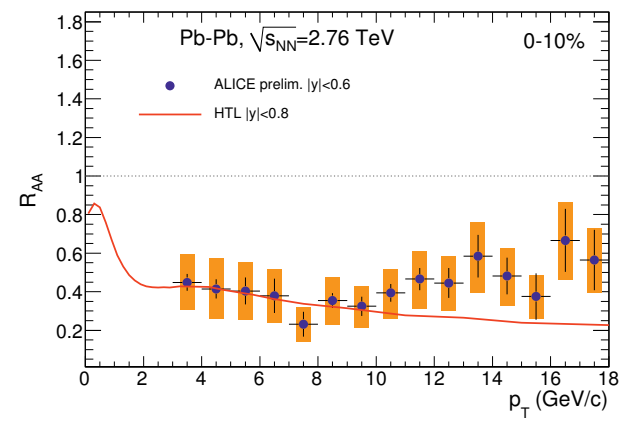

Figure 5. Predictions for heavy-flavor decay electrons from charm and beauty $\left(e_{c+b}\right)$ in $\mathrm{Pb}-\mathrm{Pb}$ collisions at the LHC compared to preliminary ALICE experimental data [25] in $0-10 \%$ most central events.

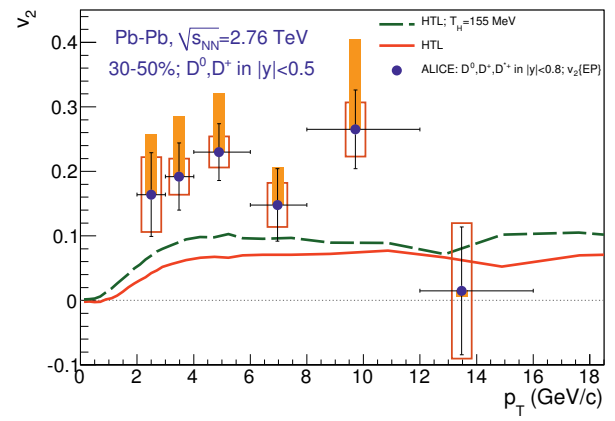

Figure 6. Elliptic flow of $D$ meson in semiperipheral (30 - 50\% centrality class) $\mathrm{Pb}-\mathrm{Pb}$ collisions at the LHC compared to ALICE data [26]. Solid line: the EoS is the same as systematically used in this work; dashed line: the phase transition occurs at $T_{c}=155 \mathrm{MeV}$.

angle of the reaction plane, which is defined by the impact parameter of the colliding nuclei. For non-central collisions, the dominant harmonic in the Fourier series is the second one, $v_{2}$, commonly called elliptic flow, which reflects the lenticular shape of the overlap region of the colliding nuclei. Non-zero elliptic flow of final state hadrons originates from the build-up of a collective motion of the medium constituents (dominant at low $p_{\mathrm{T}}$ ) and from the path-length dependence of in-medium parton energy loss.

In Fig. 6 we address the elliptic flow of $D$ mesons. Outcomes of our Langevin setup for the elliptic flow $v_{2}$ are compared to ALICE data [26] in semi-peripheral (30-50\%) Pb-Pb collisions. HTL results significantly underestimate the experimental data at low $p_{T}$, achieving at larger $p_{T}$ 's an asymptotic plateau (experimentally observed also in the case of light-hadron spectra) arising from the path-length dependence of the energy loss. 

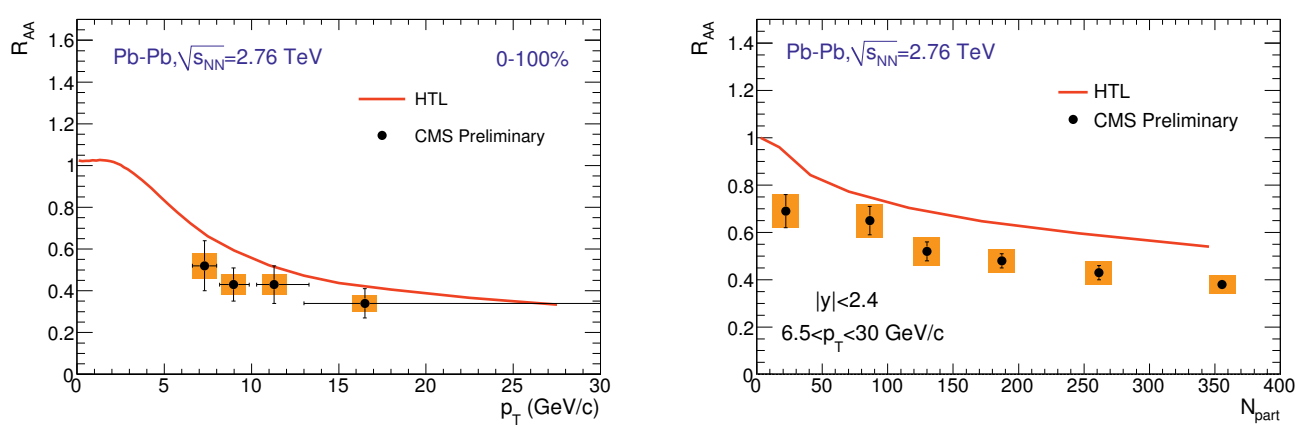

Figure 7. Left panel: $R_{A A}$ as a function of $p_{T}$ of non-prompt $J / \psi$ 's (from B decays) in minimum-bias Pb$\mathrm{Pb}$ collisions at the LHC. Results of our setup are compared to preliminary CMS data [28]. Right panel: the centrality dependence of the $R_{A A}$ of non-prompt $J / \psi$ 's.

In the equation of state (EoS), used in our calculations, of Ref. [10], the phase transition occurs with a smooth cross-over in a temperature interval between 160 and $190 \mathrm{MeV}$, with an average value around $180 \mathrm{MeV}$. Most recent lattice results [27], however, quote a significantly lower critical temperature: $155 \mathrm{MeV}$. To test the sensitivity of our results on the details of the phase transition, we have performed a calculation assuming a constant transition temperature of $155 \mathrm{MeV}$. The results for the $v_{2}$ parameters are shown in Fig. 6 (dashed line). The fact that experimental data are underestimated (although the lower transition temperature seems to be preferred) at small $p_{T}$ is a strong hint that an important contribution to the elliptic flow of $D$ mesons may come from coalescence with thermal partons at hadronization.

Finally, we apply our setup to the study of beauty dynamics in the QGP. Indirect information on beauty in heavy-ion collisions comes through the non-prompt $J / \psi$ (from $B \rightarrow J / \psi+X$ decays) measurements by CMS [28]. Our results for the displaced $J / \psi R_{A A}$, versus $p_{T}$ and centrality, are shown in Fig. 7 and compared to the CMS preliminary results. The data seem to point to a stronger quenching than predicted by theory, at variance with the charm data, whose quenching is generally overestimated at large $p_{T}$ 's. On the other hand, the minimum-bias $R_{A A}$ as a function of $p_{T}$ (left panel of Fig. 7) shows a fair agreement with the one of the HTL calculation. The better agreement, in the case of bottom quarks can be understood since the coalescence mechanism is expected to alter the shape of $R_{A A}$ much less than in the case of charm quarks. At the same time, the heavier mass of the $b$ quark should make the perturbative calculation of the transport coefficients more reliable.

\section{Conclusions and future improvements}

In this paper we have shown some results provided by our transport setup for the study of heavy quarks in the QGP. Theory outcomes have been compared to the most recent experimental data collected at RHIC and LHC. If the experimental heavy-flavor $R_{A A}$ can be reproduced reasonably well over most of the $p_{T}$ range experimentally accessible, a consistent description within the same setup of the elliptic flow of charm is still lacking.

Results for the $R_{A A}$ of non prompt $J / \psi$ 's from $B$ decays of the CMS collaboration have been compared to the outcomes of our setup, with a decent agreement. 
A few important items remain to be addressed and are left for future work. First of all, a modelling of coalescence, necessary in order to provide predictions at low $p_{T}$. Secondly, extending the setup to the forward-rapidity region, so that one can study also the rapidity dependence of the various heavyflavor observables and face also the single-muon data measured by the ALICE experiment. This step would require to interface our transport setup with the output of a full $3+1$ hydrodynamic code, which is currently under development [29]. Finally, we plan to include a rescattering of charmed and bottomed hadrons in the hadronic phase, neglected so far: this should also be very important for the building of the elliptic flow.

\section{References}

[1] A. Beraudo, A. De Pace, W.M. Alberico, A. Molinari, Nucl.Phys. A 831, 59 (2009)

[2] W.M. Alberico, A. Beraudo, A. De Pace, A. Molinari, M. Monteno, M. Nardi, F. Prino, Eur. Phys. J. C 71, 1666 (2011)

[3] W.M. Alberico, A. Beraudo, A. De Pace, A. Molinari, M. Monteno, M. Nardi, F. Prino, M. Sitta, Eur.Phys.J. C73, 2481 (2013)

[4] S. Frixione, P. Nason, G. Ridolfi, J. High Energy Phys. 0709, 126 (2007)

[5] T. Sjostrand, S. Mrenna, P.Z. Skands, J. High Energy Phys. 0605, 026 (2006)

[6] S. Alioli, P. Nason, C. Oleari, E. Re, J. High Energy Phys. 1006, 043 (2010)

[7] K.J. Eskola, H. Paukkunen, C.A. Salgado, J. High Energy Phys. 0904, 065 (2009)

[8] P.F. Kolb, J. Sollfrank, U. Heinz, Phys. Rev. C 62, 054909 (2000)

[9] P.F. Kolb, R. Rapp, Phys. Rev. C 67, 044903 (2003)

[10] P. Romatschke, U. Romatschke, Phys. Rev. Lett. 99, 172301 (2007)

[11] M. Luzum, P. Romatschke, Phys. Rev. C 78, 034915 (2008)

[12] M. Luzum, P. Romatschke, Phys. Rev. Lett. 103, 262302 (2009)

[13] ZEUS Collaboration (S. Chekanov et al.), Eur. Phys. J. C 44, 351 (2005)

[14] ALEPH Collaboration (R. Barate et al.), Eur. Phys. J. C 16, 597 (2000) 597

[15] Heavy Flavor Averaging Group Collaboration (D. Asner et al.), arXiv:1010.1589

[16] M. Cacciari, P. Nason, J. High Energy Phys. 0309, 006 (2003)

[17] M. Cacciari, S. Frixione, M.L. Mangano, P. Nason, G. Ridolfi, J. High Energy Phys. 0407, 033 (2004)

[18] ALEPH Collaboration (A. Heister et al.), Phys. Lett. B 512, 30 (2001)

[19] SLD Collaboration (K. Abe et al.), Phys. Rev. D 65, 092006 (2002); Erratum-ibid. D 66, 079905 (2002)

[20] D. Tlusty (for STAR Collaboration), Nucl. Phys. A 904-905, 639c (2013)

[21] H. van Hees, V. Greco, R. Rapp, Phys. Rev. C 73, 034913 (2006)

[22] PHENIX Collaboration (A. Adare et al.), Phys. Rev. C 84, 044905 (2011)

[23] M. Mustafa (for the STAR Collaboration), Nucl. Phys. A 904-905, 665c (2013)

[24] ALICE Collaboration (B.I. Abelev et al.), J. High energy Phys. 1209, 112 (2012)

[25] S. Sakai, (for the ALICE Collaboration), Nucl. Phys. A 904-905, 661c (2013)

[26] ALICE Collaboration (B.I. Abelev et al.), arXiv:1305.2707

[27] S. Borsanyi et al. [Wuppertal-Budapest Collaboration], JHEP 1009, 073 (2010)

[28] CMS Collaboration (S. Chatrchyan et al.), CMS-PAS-HIN-12-014

[29] L. Del Zanna, V. Chandra, G. Inghirami, V. Rolando, A. Beraudo, A. De Pace, G. Pagliara, A. Drago, F. Becattini, arXiv:1305.7052 